\title{
Effect of Magnetic Shear on Dissipative Drift Instabilities
}

P. N. Guzdar, L. Chen, P. K. Kaw, and C. Oberman

Plasma Physics Laboratory, Princeton University

Princeton, New Jersey 08540

\begin{abstract}
In this letter we report the results of a

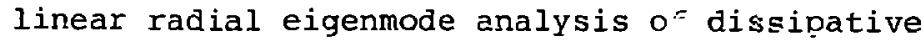
drift waves in a plasma with magnetic shear and spatially varying density gracient. The results of the analysis are shown to be consistert with a recent experiment on the study of d ssipative drift instabilities in a toroidal stelıarator.
\end{abstract}


Recently, Vujtsenya et al. 1 have reported the results of an interesting experiment on the effect of magnetic shear on drift-dissipative instabilities in a collisionally dominated plasma in a toroidal stellarator. Their results indicate that drift waves are localized near the maximum of the arift frequency $\omega_{*}$ (the variation of $\omega_{*}$ arising due to a spatially varying density gradient) and are strongly reduced in amplitude when the shear in the magnetic field exceeds a critical value. In this letter we present the results of a linear radial eigenmode analysis ${ }^{2}$ of dissipative drift waves in a plasma with magnetic shear and a spatially varying density gradient, and propose a possible interpretation of the above experiment.

The local theory of dissipative drift waves is well known (see e.g., Kadomtsev ${ }^{3}$ ); this is applicable as such only to sheariess situations and with no spatial variation of the drift frequency $\omega_{*}$. Moiseev and sagdeev ${ }^{4}$ have carried out a radial eigenmode analysis including the spatial variation of $\omega_{*}$ but with no magnetic shear. The inclusion of shear strongly modifies the r results. Further, the previous analyses 5,6 with magnetic shear have been rather qualitative and incomplete.

We consider a plane plasma slab with the density variation and magnetic shear along $x$ direction; i.e., $\mathrm{n}_{0}(\mathrm{x})=$ $\mathrm{n}_{0}(0) \exp \left[\mathrm{x} / L_{n}\left(1-x^{2} / 3 I_{*}^{2}\right)\right]$ and $\underset{\sim}{B}(x)=B_{0}\left(I_{\sim} z+I_{\sim} y / L_{s}\right)$. Here $I_{s^{\prime}} I_{n^{\prime}}$, and $I_{*}$ are the shear length, the density scale length, and the scale length for variation of density gradient, 
respectively. $x=0$ is taken as the mode-rational surface; i.e., surface on which $\underline{\mathbf{k}} \cdot \underline{\mathrm{B}}=\mathbf{0}$. For simplicity, we have assumed the density gradient to peak at $x=0$. As usual, we shall assume electron thermal conductivity parallel to the lines to be very large to justify using isothermal electrons and approximate the ions as a cold species $\left(T_{i}=0\right)$. If we use the linearized equations of continuity and motion for electrons and ions, take perturbations of the form $\phi(\underset{\sim}{x}, t)=\phi(x) \exp \left[\dot{i}\left(k_{y^{*}}-\omega t\right)\right]$ and finally assume the quasineutrality condition $n_{e} \approx n_{i}$, we obtain the eigenmode equation

$$
p_{s}^{2}\left(\frac{d^{2}}{d x^{2}}-k_{y}^{2}\right) \phi-\frac{x^{2}}{x^{2}-i x_{R}^{2}}\left[1-\frac{\omega_{*}}{\omega}\left(1-\frac{x^{2}}{L_{*}^{2}}\right)-\frac{x^{2}}{x_{s}^{2}}\right] \phi=0
$$

where $k_{\|}=k_{\|}^{\prime} x \equiv k_{y} x / L_{s}, x_{s}^{2}=\omega^{2} / k_{\|}^{2}{ }^{2} c_{s}^{2}, \omega_{*_{0}}=k_{y} \rho_{s} c_{s} / I_{n}$, $x_{R}^{2}=\omega \nu_{e i} / k_{\|}^{2} v_{e T}^{2}, v_{e T}^{2}=T_{e} / m_{e}, c_{s}^{2}=T_{e} / m_{i}, \rho_{s}^{2}=c_{s}^{2} / \omega_{c i}^{2}$ and the other symbols have obvious meanings. The first term comes from the ion polarization drift, che coefficient $x^{2} /\left(x^{2}-i x_{R}^{2}\right)$ is a consequence of the resistive parallel dynamics of electrons and the $x^{2} / x_{s}^{2}$ term has its origin in the parallel ion motion. Moiseev and Sagdeev ${ }^{4}$ solved Eq. (I) in the limit $\mathrm{x} \ll, \mathrm{x}_{\mathrm{R}}, \mathrm{k}_{\|}^{\prime} \mathrm{x}=\mathrm{k}_{\|}=$constant (no shear) and $\mathrm{x}_{\mathrm{s}}=\infty$ (ignoring the parallel ion dynamics). 
We first examine the eigenvalue problem for $L_{*}=\infty$; i.e., $\omega_{*}$ independent of $x$. After changing variables and rearranging the terms, Eg. (1) may be rewritten as

$$
\left(\frac{d^{2}}{d \xi^{2}}+\delta-\frac{\xi^{2}}{4}-\frac{\Lambda}{\xi^{2}+\xi_{R}^{2}}\right) \phi=0
$$

where $\xi=\mathrm{x} \exp (i \pi / 4) / \lambda, \lambda^{2}=\rho_{\mathrm{s}} \mathrm{x}_{\mathrm{s}} / 2, \xi_{\mathrm{R}}^{2}=\mathrm{x}_{\mathrm{R}}^{2} / \lambda^{2}$,

$\delta=i\left(\lambda^{2} / \rho_{s}^{2}\right)\left[1-\omega_{*} / \omega+k_{Y}^{2} \rho_{s}^{2}-i\left(x_{R}^{2} / x_{s}^{2}\right)\right]$ and

$\Lambda=i\left(x_{R}^{2} / \rho_{s}^{2}\right)\left[1-\omega_{*} / \omega-i\left(x_{R}^{2} / x_{s}^{2}\right)\right]$. When $\xi_{R}^{2}=2\left(m_{e} / m_{i}\right)\left(\nu_{e i} / \omega_{*}\right)$

$\left(I_{s} / L_{n}\right) \ll 1$, the eigenvalue problem associated with Eq.

may be solved by the following matching procedure. In the outer region $\xi \gg \xi_{R}\left[\right.$ so $\xi_{R}$ may be neglected in Eq. (2)] and the solution which properly decays away for $x \rightarrow \infty$ and $\operatorname{Im} \omega>0$ is given by

$$
\phi_{0}=A_{0}\left(\xi^{2} / 2\right)^{(2 \alpha+1) / 4} \cup\left(a, b, \xi^{2} / 2\right) \exp \left(-\xi^{2} / 4\right)
$$

where $U$ is Kummer's confluent hypergeometric function ${ }^{7}$ and $\alpha=-(1 / 2)(1+4 \Lambda)^{1 / 2}, b=1+\alpha, a=(1 / 2)(b-\delta)$. In the inner region $\xi \sim \xi_{\mathrm{R}} \ll 1$ and the solution may be approximated as

$$
\phi_{I}=\left(1+\xi^{2} / \xi_{R}^{2}\right)^{1 / 2}\left[\mathrm{~A}_{I} P_{V}^{1}\left(\xi / i \xi_{R}\right)+{ }_{\left.B_{I} Q_{\nu}^{1}\left(\xi / i \xi_{R}\right)\right]}\right.
$$


where the $\mathrm{P}_{v}^{1}$ and $\mathrm{Q}_{v}^{1}$ are the associated Legendre functions and the order $v=-(I / 2+\alpha)$. The constants $B_{I}$ and $A_{I}$ are related by the parity condition at the origin, viz., $\phi_{I}(0)=0$ for odd modes and $d \phi_{I} /\left.d \xi\right|_{\xi=0}=0$ for even modes. Matching the outer region solution $\phi_{0}$ with $\xi \ll 1$ to the asymptotic form of the inner region solution (for $1 \gg \xi \gg \xi_{R}$ ), one gets the eigenvalue condition

$$
\begin{aligned}
\frac{\Gamma(a)}{\Gamma[a+\nu+(1 / 2)]}= & \left(-i \frac{\xi_{R}}{2^{3 / 2}}\right)^{1+2 v} \frac{\Gamma(v) \Gamma[-(1 / 2)-v] \Gamma[(1 / 2)-v]}{\Gamma(-1-v) \Gamma[(1 / 2)+v] \Gamma[(3 / 2)+v]} \\
& \left(1+\pi \frac{B_{I}}{A_{I}} \cot \pi \nu\right)
\end{aligned}
$$

where $\Gamma$ denotes the usual gamma function. Note the definitions of $v, a$, and other related quantities following Eqs. (4), (3), and (2), respectively.

The eigenvalue condition (5) is, in general, difficult to solve analytically for the complex frequency $\omega$. However, approximate results may be obtained in certain interesting 1 imits. When $\mathrm{k}^{2} \rho_{\mathrm{s}}^{2} \ll \mathrm{L}_{\mathrm{n}} / \mathrm{L}_{\mathrm{s}}$. [which may be rewritten as $\left.|\Lambda| \simeq k^{2} \rho_{s}^{2}\left(L_{s} / I_{n}\right)\left|\xi_{R}^{2}\right| \ll 1\right]$ one finds $v \simeq \Lambda$. Evaluating $B_{I} / A_{I}$ for even parity modes, one obtains the approximate eigenvalue condition 


$$
a \simeq-n+(-1)^{n+1} 2^{-3 / 2} \frac{\pi \Lambda}{\xi_{R} \Gamma[(1 / 2)-n]} ; n=0,1, \ldots
$$

For the lowest mode we then obtain the dispersion relation

$$
1-\frac{\omega_{*}}{\omega} \cong-\left(i \frac{I_{n}}{I_{s}}+k_{y}^{2} p_{s}^{2}\right)\left[1+\left(\pi \frac{v_{e i}}{\omega_{*}} \frac{m_{e}}{m_{i}} \frac{L_{s}}{L_{n}}\right)^{1 / 2}\right] .
$$

Equation (6) leads to the surprising result that in a sheared geometry, resistivity only enhances the shear damping of drift waves by a factor proportional to $v^{1 / 2}$ and that no unstable mode exists. A detailed analysis ${ }^{2}$ shows that the expected growth terms are propcrtional to $v_{e i}$ and are always subdominant to the resistivity induced enhancement of shear damping. For $\mathrm{k}^{2} \rho_{\mathrm{s}}^{2} \gg I_{\mathrm{n}} / I_{\mathrm{s}}$, the other simple and instructive limit $|\Lambda| \gg 1$ can be used. Here again one finds for the lowest mode $v \simeq \Lambda^{1 / 2}-1 / 2, a \simeq-\Lambda^{1 / 2}$ and the approximate dispersion relation

$I-\frac{\omega_{*}}{\omega} \cong-k_{y} \rho_{s}\left[k_{y} \rho_{s}+(1+i)\left(2 \frac{m_{e}}{m_{i}} \frac{v_{e i}}{w_{*}}\right)^{1 / 2}\right]$.

Even in this limit, no unstable eigenmodes exist. 
Our analysis thus leads us to the surprising result that for $\left(m_{e} \nu_{e i} L_{s} / m_{i} \omega_{*} L_{n}\right) \ll 1$, electrostatic drift waves in a plane plasma slab with magnetic shear only exist as damped eigenmodes. We have verified this result by a direct numerical integration of the differential Eq. (2) using a shooting method and also by a complex WKB treatment. The numerical calculations show that the modes are stable even in the range $\left(m_{e}{ }^{\nu} i^{I} s^{\prime} / m_{i} \omega_{*} L_{n}\right)>1$. Details of these investigations will be separately published. ${ }^{2}$ None of the above investigations, however, forbids a convective amplification of wave-packets of drift waves. ${ }^{8}$ Physically the eigenmodes are damped because of a resistivity induced enhancement of shear damping. An obvious way to recover growing eigenmodes is thus to make shear damping ineffective. This may be done by introducing a proper profile for $\omega_{*}$, i.e., by discussing the case with $L_{n:} \neq \infty$.

Equation (1) may be rewritten as

$$
\left(\frac{d^{2}}{d \eta^{2}}+\delta_{0}-\frac{\eta^{2}}{4}-\frac{\Lambda_{0}}{\eta^{2}-i \eta_{R}^{2}}\right) \phi(n)=0
$$

where $\eta=x / \lambda_{0}, \lambda_{0}^{2}=x_{0} \rho_{s} / 2, x_{0}^{-2}=\left(\omega_{*_{0}} / \omega\right) L_{\star}^{-2}-x_{s}^{-2}, \eta_{R}=x_{R} / \lambda_{0}$, $\delta_{0}=-\left(\lambda_{0} / \rho_{s}\right)^{2}\left[1-\left(\omega_{*_{0}} / \omega\right)+k_{Y}^{2} \rho_{s}^{2}+i\left(x_{R}^{2} / x_{o}^{2}\right)\right]$, $\Lambda_{0}=i\left(x_{R} / \rho_{S}\right)^{2}\left[1-\left(\omega_{*_{0}} / \omega\right)+i\left(x_{R}^{2} / x_{0}^{2}\right)\right]$. To nullify shear 
damping, we must require that $\mathrm{R}_{\mathrm{e}}\left(\mathrm{x}_{0}^{-2}\right)>0$; i.e.,

$$
I_{*}^{2}<x_{s}^{2}\left(\omega_{*_{0}} / \omega\right)
$$

For $\left|n_{R}\right|^{2} \ll 1$, we can use the same matching procedure as above. The analogue of $\mathrm{Eq}$. (6) is

$$
1-\frac{\omega_{*_{0}}}{\omega}=-\left(k_{y}^{2} \rho_{s}^{2}+\frac{\rho_{s}}{x_{0}}\right)\left[1+\frac{\pi^{1 / 2}}{2}(I-i)\left(\frac{\nu_{e i}}{\omega_{*_{0}}} \frac{m_{e}}{m_{i}} \frac{L_{s}^{2}}{L_{n}^{2}} \frac{\rho_{s}}{x_{0}}\right)^{1 / 2}\right] .
$$

Equation (10) shows that shear damping is absent and that resistivity contributes to growth of the eigenmode. For shorter wavelengths, the analogue of Eq. (7) takes the form

$1-\frac{\omega_{*_{0}}}{\omega} \simeq-k_{Y}^{2} \rho_{s}^{2}+(i-1) k_{Y} \rho_{s}\left(\frac{\rho_{s} L_{s}}{x_{0} I_{n}}\right)\left(\frac{\nu_{e i}}{\omega_{*_{0}}} \frac{m_{e}}{m_{i}}\right)^{1 / 2}$

which again shows an eigenmode growth rate $\propto \nu_{\mathrm{e}}^{1 / 2}$. It is clear that inequality (9) is the critical condition for the existence of growing eigenmodes. Wien it is not obeyed, we again revert to the previous case of shear damping only. 
The experiment of Vojtsenya et al. ${ }^{1}$ was done on an argon plasma in a stellarator with typical parameters: $\mathrm{T}_{\mathrm{e}} \approx 6 \mathrm{eV}$, $\mathrm{n}_{\mathrm{e}} \approx 2 \times 10^{11} \mathrm{~cm}^{-3}, \mathrm{~T}_{\mathrm{e}} \gg \mathrm{T}_{i}, \mathrm{~B}_{\mathrm{o}}=3.1 \mathrm{kilogauss}$. This justifies ${ }^{3}$ the use of electrostatic drift wave model to describe their experiment, since $B=8 \pi n T / B_{0}^{2}<\left(m_{e} / m_{i}\right)$. Our assumption of cold ions is also obviously good. The experiment demonstrated the excitation of dissipative drift waves $\left(\omega \sim \omega_{*}<v_{e i}\right)$ localized in the neighborhood of a maximum of $\omega_{*}$. It was also discovered that when the shear parameter $\theta=I_{n} / I_{s}>0.05$, a substantial reduction in the level of drift wave oscillations takes place. We now propose that the transition from a growing eigenmode to a stable one, may lead to a similar reduction in the oscillation level. In the former case, the instability keeps growing until nonlinear effects saturate the waves. In the latter case, there is only convective amplification of waves and they may saturate even at low levels, by simply convecting out of the unstable region. In the experiment, the transition can occur because inequality switches as $\theta=I_{n} / L_{s}$ is increased. The condition of shear stabilization of density gradient localized drift waves may thus be written $\left(L_{\star} / \rho_{s}\right)>\Delta \theta^{-1}$, where $\Delta$ is a numerical factor taking account of the fact that (i) the experimental density gradient variation is not parabolic, (ii) the transition to a low saturation convective mode does not occur exactly at (9), and (iii) inaccuracies in our estimates of various parameters. 
For an $L_{*} \cong 2 \mathrm{cms}$ and the other experimental parameters given above, we find agreement with the experimental value of $\theta_{C}=0.05$, if we choose $\Delta \sim 1 / 5$. Considering the above uncertainties, this seems pretty reasonable.

\section{ACKNOWLEDGMENTS}

This work was partly supported by United States Department of Energy Contract No. EY-76-C-02-3073 and by United States Air Force office of Scientific Research Contract No. F 44620-75-C-0037. 
REFERENCES

Iv. S. Vojtsenya, A. Yu. Voloshko, V. M. Zalkind, S. I. Solodovchenko, V. P. Tarasenko, A. F. Stan, Nucl. Fusion 17, 651 (1977).

2 L. Chen, P. N. Guzdar, J. Y. Hsu, P. K. Kaw, C. Oberman, and R. White, Plasma Physics Laboratory, Princetor University, Rept. No. PPPI-1423.

3 B. B. Kadomtsey, Plasma Turbulence, (A.cademic Press), p. 88, (1965).

${ }^{4}$ S. S. Moiseev and R. Z, Sagdeev, Sov. Phys. Tech. Phys. 9, 196 (1964) [zh. Tekh. Fiz. 34, 248 (1964)].

5. A. Rukhadze and V. P. Silin, Sov. Phys. Usp. 11, 659 (1969) [Usp. Fiz. Nauk. 95, 87 (1968)].

${ }^{6}$ B. B. Kadomtsev and O. P. Pogutse, Reviews of Plasma Phys., (Consultants Bureau) 5, 2.49 (1970).

7M. Abramowitz and I. A. Stegun, N. B. S., Appl. Math. Series 5 503 (1964).

8. Coppi, G. Laval, R. Pellat, and M. N. Rosenbluth, Nucl. Fusion 6, 261 (1966). 